\title{
Quantifying the effects of mechanical signals on musculoskeletal quality in a model of complete estrogen deprivation
}

\author{
Reid Wilson ${ }^{1}$, Gabriel M. Pagnotti, PhD ${ }^{1}$, Khalid S. Mohammad, MD, PhD ${ }^{1}$ \\ Theresa A. Guise, MD ${ }^{1}$ \\ ${ }^{1}$ Indiana University; Department of Medicine, Endocrinology
}

\section{Background and Hypothesis:}

Post-menopausal, estrogen-receptor positive breast cancer patients are treated with aromatase inhibitors (Als) to limit tumor progression; however, this causes adverse musculoskeletal effects. Zoledronic acid (ZA) is prescribed to inhibit bone resorption. Mechanical signals, delivered via low intensity vibration (LIV), stimulate bone formation. We hypothesize that combining LIV with ZA will mitigate bone loss in a murine model of complete estrogen-deprivation more effectively than either treatment alone.

\section{Project Methods:}

21-week-old C57BL/6 mice ( $\mathrm{n}=20 /$ group) were ovariectomized, receiving daily letrozole injections (OVX/AI) with LIV (OVX/AI+LIV), ZA (OVXIAI+ZA), LIV and ZA (OVX/AI+LIVIZA) or underwent sham surgery with daily PBS (vehicle) injections (SH-OVX) for 22 weeks. Longitudinal dual energy $\mathrm{X}$-ray absorptiometry (DEXA) and micro-computed tomography scans were analyzed for changes in body composition and bone microarchitecture, respectively.

\section{Results:}

OVX/AI reduced whole body $(p<0.0001)$ and lumbar spine $(p<0.0001)$ bone mineral density (BMD) as compared to SH-OVX by $11 \%$ and $28 \%$, respectively, as measured via DEXA. At 22w, OVX/AI+LIV/ZA had greater BMD across both regions-of-interest relative to OVX/AI $(p<0.0001)$. Cortical bone area fraction $(p<0.0001)$ and cortical thickness $(p \leq 0.001)$ in distal femora decreased by $8 \%$ and $9 \%$, respectively, in OVX/AI relative to SH-OVX. In OVX/AI+LIVIZA, these parameters were greater relative to OVX/AI $(p \leq 0.0001)$. OVX/AI+ZA and OVX/AI+LIV groups were not significantly different from OVX/AI in either cortical bone area fraction or cortical thickness.

\section{Conclusion and Potential Impact:}

Complete estrogen-deprivation reduced bone mineral density and altered microarchitecture. Mechanical signals provided via LIV combined with ZA prevent bone loss in aged estrogen-deprived mice. Combining these treatment strategies may reduce skeletal morbidity in breast cancer patients. 